\title{
Immunohistochemical Biomarkers in Gastric Cancer Research and Management
}

\author{
Elena Lastraioli, Maria Raffaella Romoli, and Annarosa Arcangeli \\ Department of Experimental Pathology and Oncology, University of Florence, 50134 Florence, Italy \\ Correspondence should be addressed to Annarosa Arcangeli, annarosa.arcangeli@unifi.it \\ Received 16 February 2012; Revised 22 April 2012; Accepted 25 April 2012 \\ Academic Editor: Marco Bernini
}

Copyright (c) 2012 Elena Lastraioli et al. This is an open access article distributed under the Creative Commons Attribution License, which permits unrestricted use, distribution, and reproduction in any medium, provided the original work is properly cited.

\begin{abstract}
Gastric cancer still represents a major health problem, despite a decrease in its incidence in the last years. Due to the social impact of gastric cancer (GC), there is a need for novel biomarkers in order to stratify patients into appropriate screening, surveillance, or treatment programs. Although histopathology remains the most reliable and less expensive method, numerous efforts have been made searching for novel biomarkers. In recent years, several molecules have been identified and tested for their clinical relevance in GC management. In this paper, we will focus on a well-known GC marker, whose determination is mandatory in GC, HER2, a marker whose correlation with prognosis is still controversial (VEGF-A) and a quite novel, unconventional marker, the ether-àgo-go-related gene 1 (hERG1). All these proteins can be easily detected with immunohistochemistry, a technique widely used both in diagnostic and research laboratories that represents a link between surgical and molecular pathology, basic science, and clinical medicine.
\end{abstract}

\section{Gastric Cancer}

Gastric cancer (GC) still represents a major health problem, despite a decrease in its incidence in the last years [1]. According to the most recent estimates, GC accounts for $8 \%$ of the total cancer cases and for $10 \%$ of the deaths for all cancers [2]. GC is characterized by a clear geographical distribution, with over $70 \%$ of the cases occurring in developing countries. This is partly due to dietary habits as well as Helicobacter pylori infection prevalence. Indeed, the reasons accounting for the decreased GC incidence in most countries are related to changes in dietary habits, amelioration of food preservation, reduction in $H$. pylori chronic infection [3-5] as well as reduction in smoking [1].

The majority of stomach tumors are sporadic, while only a small percentage have a familial component, with an autosomal pattern of inheritance. GC is a multifactorial disease characterized by both genetic and environmental components. In sporadic cancers of the stomach, the environmental component seems to be predominant. Conversely, the genetic component plays a major role in familial cancers. About $90 \%$ of GCs are classified as adenocarcinomas, whilst the remaining $10 \%$ is represented by non-Hodgkin lymphomas, leiomyosarcomas, squamous cell carcinomas, and undifferentiated carcinomas. In this paper, we will mainly refer to adenocarcinomas, addressing them as simply "GCs." According to the Lauren's classification, two subtypes of GC can be distinguished basing on their different histology: the intestinal (I-GC) and diffuse (D-GC) types [6]. The two GC types also display different biological and etiological characteristics. Tumor cells of I-GC form glandular-like structures, a feature which lacks in D-GC, which, on the contrary, is characterized by the infiltration and thickening of the gastric wall by tumor cells. The two histological subtypes are the result of distinct pathogenetic pathways, well described in the two models, proposed to depict the pathogenesis of I-GC [7] and D-GC [8]. As shown in Figure 1, I-GC occurrence is preceded by the development of chronic gastritis, which in turn leads to atrophy, and by the subsequent appearance of intestinal metaplasia. Intestinal metaplasia arises from the proliferation of gastric stem cells, whose progeny differentiates into "intestinal type" cells (columnar, goblet, and Paneth cells), due to the persistent irritation of the gastric mucosa, caused by H. pylori [9]. 


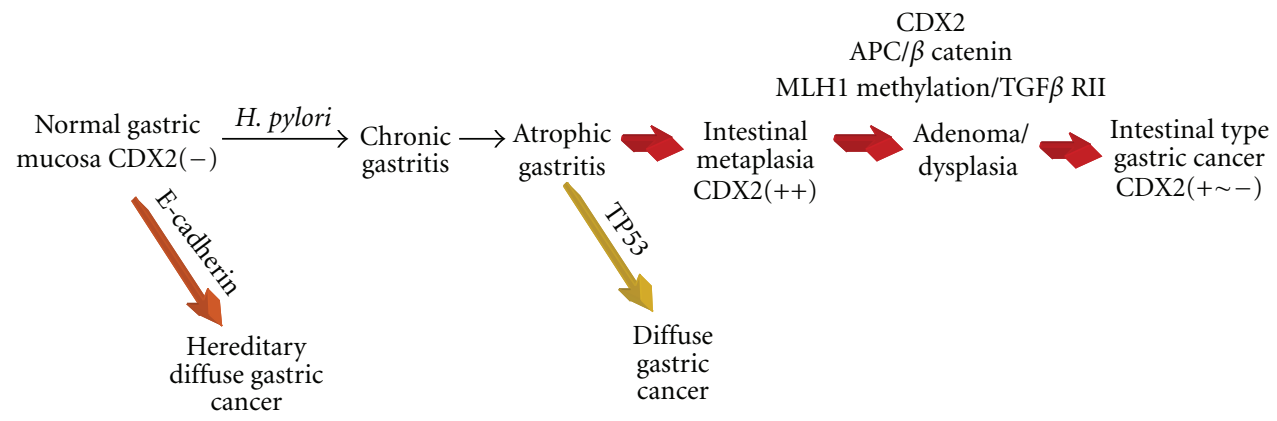

FIgURE 1: Correa model for intestinal type GC.

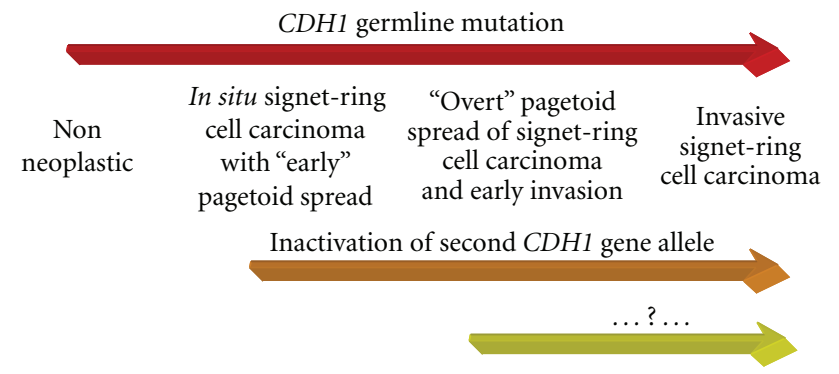

Figure 2: Carneiro's model for diffuse type GC.

The Correa model is not applicable to the pathogenesis of D-GC. The latter is, however, well described by the Carneiro model [8] (Figure 2).

The diffuse type GC is characterized by reduced or abnormal E-cadherin expression $[10,11]$. The inactivation of the second CDH1 allele (e.g., the gene encoding Ecadherin) leads to the appearance of an in situ carcinoma, with the presence of signet-ring cells with a "Pagetoid" pattern of diffusion, which is subsequently followed by the invasion of surrounding tissues. According to this model, the intraepithelial presence of signet-ring cells does not represent a secondary colonization. On the whole, Ecadherin loss/abnormality represents an early event in the cancerogenesis of D-GC [8], and the dysregulation of the gene is one of the most frequent genetic alterations in diffuse type GC [12].

Due to the social impact of GC, there is a need to stratify patients into appropriate screening, surveillance, or treatment programs. Although histopathology remains the most reliable and less expensive method, numerous efforts have been made to identify and validate novel biomarkers to accomplish the above goals. In recent years, several molecules have been identified and tested for their clinical relevance in GC management. Table 1 shows an overview of some of the biomarkers reported so far, along with the most correlated clinical parameters. With the exception of HER2, none of the biomarkers reported in the table is currently used in clinical practice, and some of them were described in single studies.

Immunohistochemistry (IHC) staining of formalin-fixed and paraffin-embedded tissues is widely used in diagnostic

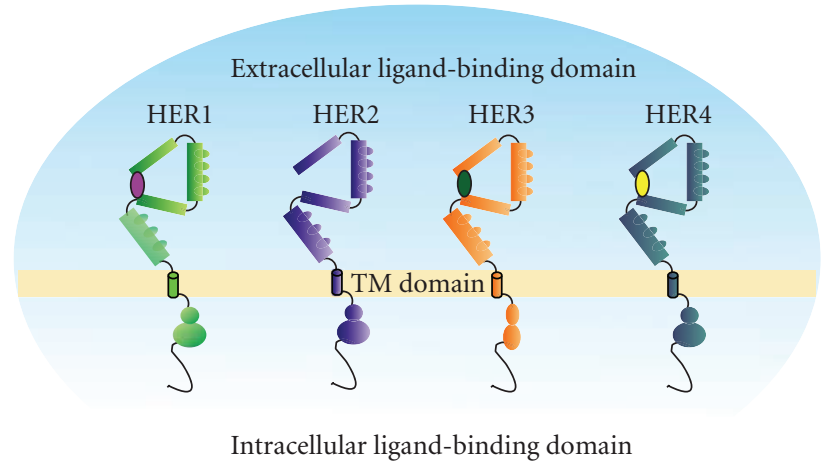

FIGURE 3: HER family receptors.

surgical pathology to gather additional information embracing those obtained with classical hematoxylin and eosin staining. IHC assists the pathologists in areas of tumor classification, multilineage differentiation, molecular correlates, and infectious etiologies. Moreover, IHC is commonly used to detect markers, which in turn can provide information on the biological behaviour and prognosis of a tumor. Different biomarkers detected by IHC are now a common component for many institutional review board protocols, for a more precise risk stratification and target identification. Therefore, IHC represents a link between surgical and molecular pathology, basic science and clinical medicine, surgery, and radiology [13].

As evidenced in Table 1, the number of potential biomarkers in GC is quite high and still increasing. Those which are easily detectable and quantifiable through IHC will be the object of the present review. In particular, we will focus on HER2, a well-known GC marker, whose determination is mandatory in GC, a marker whose correlation with prognosis is still controversial, VEGFs and quite novel, unconventional marker, hERG1.

1.1. HER2. The HER family comprises four different receptors: HER1 (EGFR or ErbB1), HER2 (ErbB2 or HER-2/Neu), HER3 (ErbB3), and HER4 (ErbB4) (Figure 3).

These receptors cooperate in the regulation of different processes, such as cell proliferation, differentiation, and 
survival [14]. HER family members are implicated in the development of different kinds of tumors and are now recognized targets for biological therapy in breast, colorectal, lung, head and neck, gastric and gastro-oesophageal junction cancer (reviewed in [15]). Upon ligand binding, the receptors dimerize, become phosphorylated, and transduce intracellular signals, that ultimately regulate the abovementioned cellular processes. Receptor dimerization can also occur through the process of receptor pairing, other than ligand binding [16]. Indeed, HER receptors can either homo- or hetero-dimerize with other HER family members, allowing multiple receptor combinations $[16,17]$.

Dimer formation leads to the phosphorylation of key intracellular proteins, that provide docking sites for a variety of subsiding signalling molecules. The latter then transmit signals to different downstream cascades, including the MAPK and the PI3K/AKT pathways [16, 18].

The HER2 gene has been recognized as a key regulator in the development of different types of tumors in particular breast cancer [60]. In GC, HER2 acts as an oncogene, since gene amplification reflects in protein overexpression, therefore giving selective advantage to malignant cells. In GC, HER2 overexpression has been correlated with poor outcome and a more aggressive disease [20] as well as with shorter survival [19-22, 61-65]. Based on data presented at the ASCO meeting in 2009 , about $22 \%$ of patients with advanced GC have tumors which overexpress HER2. In a large multicentric trial carried out on GC patients (ToGA study), a survival benefit of trastuzumab (herceptin) treatment in HER2-positive patients (IHC score $3+$ ) has been shown [23]. Therefore, HER2 represents a promising therapeutic target. However the optimal HER2 testing strategy has not been defined yet. Due to the recent approval of trastuzumab for HER2-positive GC in Europe, HER2 diagnostics is now mandatory: IHC is used as primary test, and it is followed by fluorescence in situ hybridization (FISH) in IHC2+ cases [27]. A more recent paper [26] showed that HER2 amplification can be detected in the two components (intestinal type and diffuse type areas of the neoplastic lesion) of Lauren mixed-type tumors. Standardization of HER2 testing procedures and interpretation is, therefore, an essential step to ensure accurate and reproducible results. This point acquires even more relevance, since it has been shown that HER2 status determination through the same protocol used for breast cancer, might lead to significant loss of patients [66] as there are important and significant differences in HER2 status determination between the two types of cancer (Table 2, see also [67]). As reported in Table 2, samples are given a score according to the intensity, degree of membrane reactivity and the percentage of immunoreactive cells. Scores 0 and $1+$ are considered as negative, score $3+$ is considered as positive, while $2+$ samples are considered as equivocal and should be retested by fluorescence in situ hybridization (FISH) and chromogenic in situ hybridization (CISH).

If FISH is used as first screening step, only few IHC3+ cases may be missed but it might be found a high percentage of nonresponders according to ToGA results [24, 68]. In a more recent study published in 2011 [69] it was shown
TABLE 1: Immunohistochemical markers in GC

\begin{tabular}{|c|c|c|}
\hline IHC marker & Parameter & Reference \\
\hline \multirow{4}{*}{ HER2 } & Prognosis & [19-23] \\
\hline & Therapeutic response & {$[24]$} \\
\hline & Lymph node metastasis & {$[25]$} \\
\hline & Lauren histotype & {$[26,27]$} \\
\hline \multirow{4}{*}{ VEGF } & Prognosis & {$[28-31]$} \\
\hline & Lauren histotype & [32] \\
\hline & Tumor progression & {$[33]$} \\
\hline & Therapeutic response & {$[34]$} \\
\hline hERG1 & Prognosis & {$[35]$} \\
\hline \multirow{4}{*}{ KLF5 } & Grading & {$[36]$} \\
\hline & Stage & {$[36,37]$} \\
\hline & Lymph node status & {$[36,37]$} \\
\hline & Prognosis & {$[36,37]$} \\
\hline \multirow{2}{*}{ CA IX } & Lymph node metastasis & {$[38]$} \\
\hline & Prognosis & {$[38]$} \\
\hline Ki67 & Lymph node metastasis & {$[25]$} \\
\hline \multirow{2}{*}{ PKP3 } & Stage & {$[39]$} \\
\hline & Prognosis & [39] \\
\hline MMP-2 & Prognosis & {$[29,31]$} \\
\hline HDAC & Prognosis & {$[40-42]$} \\
\hline$B c l-2$ & Lymph node metastasis & [25] \\
\hline $\mathrm{Bcl}-6$ & Prognosis & {$[43]$} \\
\hline \multirow{3}{*}{ SATB1 } & Lymph node metastasis & {$[44,45]$} \\
\hline & Distant metastasis & {$[44,45]$} \\
\hline & Stage & {$[44,45]$} \\
\hline$c-m y c 2$ & Lymph node metastasis & {$[25]$} \\
\hline TGF $\beta$ & Stage & {$[46]$} \\
\hline \multirow{4}{*}{ E-cadherin } & Prognosis & {$[31,47-52]$} \\
\hline & Invasion & {$[53]$} \\
\hline & Grading & {$[54,55]$} \\
\hline & Lauren histotype & {$[54]$} \\
\hline COX-2 & Prognosis & {$[56]$} \\
\hline TSP-1 & Prognosis & [57] \\
\hline Bax & Prognosis & {$[58,59]$} \\
\hline
\end{tabular}

that HER2 testing in GC could be performed using standard breast cancer procedures and the American Society of Clinical Oncology/College of American Pathologists scoring criteria, while a group from Korea concluded that a GCspecific scoring system should be used [70].

In contrast to HER2 and despite supportive preclinical data, observed clinical success with anti-HER1 inhibitors and endocrine therapy combinations in breast cancer has been limited $[71,72]$. In addition to HER1 and HER2, there is growing interest in HER3 as a potential therapeutic target [73]. Recently, HER3 and its physiologic ligand heregulin (HRG) have been implicated in the development of resistance to antiestrogen therapies in breast carcinoma [74]. Similarly, the dual HER1 and HER2 TKI lapatinib has clinical activity and is approved for the therapy of patients 
TABLE 2: HER2 testing by immunohistochemistry in gastric cancer.

\begin{tabular}{lcc}
\hline $\begin{array}{l}\text { IHC parameters for HER2 testing } \\
\text { protocol }\end{array}$ & IHC score & Classification \\
\hline Intensity of reactivity & 0 & Negative \\
$\quad$ Absent & $1+$ & Negative \\
$\quad$ Faint & $2+$ & Equivocal* \\
$\quad$ Weak to moderate & $3+$ & Positive \\
$\quad$ Moderate to strong & $2+$ & Equivocal* \\
\hline Degree of membrane reactivity & $3+$ & Positive \\
Complete & 0 & Negative \\
& $1+$ & Negative \\
$\quad$ Incomplete & & \\
& & \\
\hline Percentage of immunoreactive cells & $1+$ & Negative \\
(membrane reactivity) & $2+$ & Equivocal* \\
& $3+$ & Positive \\
& & \\
& 0 & Negative \\
\hline
\end{tabular}

${ }^{*}$ Samples scored IHC $2+$ should be retested with fluorescence in situ hybridization (FISH) or chromogenic in situ hybridization (CISH).

whose disease has progressed on trastuzumab [74]. Pertuzumab is a recombinant humanized monoclonal antibody directed against the dimerization domain II of HER2 that is required for ligand-dependent dimerization with HER3 [74]. While trastuzumab prevents ligand-independent HER2 signaling, pertuzumab interferes with ligand-dependent HER3mediated signaling.

1.2. VEGF. The vascular endothelial growth factor (VEGF) family is a multifunctional growth factors' family, involved in processes such as angiogenesis, inflammation, and vascular regeneration. The family includes different members: VEGFA, VEGF-B, VEGF-C, VEGF-D, VEGF-E, and PlGF, characterized by the different ability to bind to three main receptors Flt1, KDR, and Flt4 (Figure 4).

It has long been known that VEGF-A is the key regulator of tumor angiogenesis [75], a complex process with a clear relevance to tumor progression and metastasis. The maximum diameter a tumor can reach without developing a new vascular network is about 1-2 mm. Hypoxia within tumor mass induces VEGF-A secretion and increased VEGFR-2 expression [76].

It has been demonstrated that VEGF-A expression is higher in I-GC compared to D-GC [32]. The expression of VEGF-C, whose main role is that of promoting lymphangiogenesis, is related to lymph node metastasis in GC [77]. VEGF expression is mirrored by microvessel density (MVD), and they are both hallmarks of enhanced angiogenesis within the tumor mass and are therefore useful tools for GC management [78]. MVD has been investigated as a promoting factor for angiogenesis with conflicting results about its relation to survival in GC. VEGF secretion promotes endothelial cell proliferation and therefore the establishment of a new vascular network. The evaluation of MVD reflects this latter process since it is evaluated by IHC with anti-CD34 or anti-CD31 antibodies which specifically indicate new formed vessels. MVD was significantly related to the T stage, as to the TNM classification, while VEGF$\mathrm{C}$ expression was significantly higher in N-positive patients [79]. No relation was found between MVD and VEGF-C expression, but VEGF-C and MVD turned out to be related to clinicopathological features [79].

Although the VEGF superfamily has been identified to critically influence tumor-related angiogenesis, the prognostic significance of VEGF expression in GC is still controversial. In particular, VEGF-A expression seems to be a negative prognostic factor, at least in EGCs [80]. Moreover, VEGF-A expression has been proven to be relevant to therapeutic response in GC patients treated with fluorouracil alone or together with cisplatin [81]. We contributed to this discussion showing that the IHC expression of VEGFA, which positively correlated with the Lauren's intestinal histotype, has a positive impact on overall survival in univariate analysis (manuscript in preparation). The reasons of the different conclusions drawn by several groups might be related to the design of the study and sample characteristics as well as geographical differences, keeping in mind that GC is a complex disease with striking differences in different countries. Furthermore, a recent paper [33], in which the impact of VEGF-A/C/D on tumor dissemination and survival in GC was evaluated, led to conclude that VEGF-D, being associated with progressive disease, could be a helpful marker of disseminated disease. The authors concluded that the targeting of VEGF-D might be therefore a potential therapeutic strategy.

Besides controversies in the interpretation of IHC data, the Avastin in Gastric Cancer (AVAGAST) trial started in 2007. It was a multinational, randomized, and placebocontrolled trial aimed to evaluate the efficacy of adding bevacizumab to capecitabine-cisplatin in the first-line treatment of advanced gastric cancer. Although AVAGAST did not reach its primary objective, adding bevacizumab to chemotherapy was associated with significant increases in progression-free survival and overall response rate in the first-line treatment of advanced gastric cancer [34].

1.3. hERG1. The human ether-à-go-go-related gene 1 ( $h E R G 1$ ) encodes for a protein, hERG1, which is functionally a voltage-dependent potassium channel $\left(\mathrm{K}_{\mathrm{V}}\right)$, with outward rectifying characteristics. hERG1 has the typical structure of $\mathrm{K}_{\mathrm{V}} \mathrm{s}$ : it is composed of four subunits, each of which formed by six transmembrane segments (S1-S6), which are assembled to form a tetramer surrounding a central aqueous pore. The S4 segment of each subunit is composed of basic aminoacids (Lys and Arg) and represents the voltage sensor [82] (Figure 5).

hERG1 constitutes the molecular basis of the cardiac rapid repolarizing current ( $\mathrm{IKr}$ ) (reviewed in [83]) and is therefore physiologically relevant to regulate the cardiac action potential [83]. In addition, hERG1 was found to be over- and mis-expressed in a wide variety of human cancers 


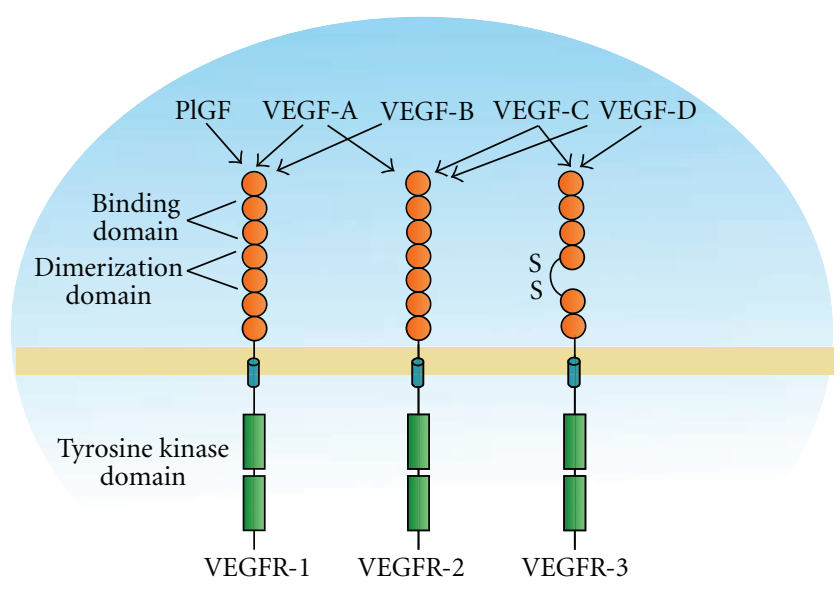

FIGURE 4: VEGF receptors and their ligands.

[84-93], where its activity is relevant to drive tumor progression. In particular, hERG1 activity is modulated by hypoxia [94] and regulates VEGF-A secretion in astrocytomas [84]. In addition, hERG1 channels mediate VEGF-Receptor-1 (FLT1)-induced cell migration and signalling in acute myeloid leukemias [90]. hERG1 is also overexpressed in cancers of the gastrointestinal tract, in particular in colorectal [91, 92] and oesophageal adenocarcinomas [93]. In colorectal cancer, it has been recently demonstrated that the IHC positivity to hERG1, in conjunction to lack of expression of the glucose transporter 1 (Glut-1) is an independent negative prognostic factor in TNM stages I and II colorectal cancers [91].

A few papers addressing the expression and role of hERG1 in GC have been published so far. hERG1 channels are expressed in GC cell lines, where their activity regulates cell proliferation in vitro $[95,96]$. Shao and colleagues [95] demonstrated that cisapride, a specific blocker of hERG1, can inhibit the growth of GC cells, by altering cell distribution within the cell cycle and inducing apoptosis. A more recent paper by the same group [97] demonstrated the correlation between hERG1 expression and tumor grading and TNM stage. Furthermore, inhibition of the channel with specific siRNAs resulted in a reduction of tumor growth and colony formation. Based on these results, hERG1 protein could be considered as a potential therapeutic target. More recently, Ding and colleagues [35] demonstrated significant differences in hERG1 protein expression, according to factors such as serosal invasion, venous invasion, and TNM stage. The mean survival time for hERG1 positive patients was significantly shorter than that of hERG1 negative ones and hERG1 expression was proven to be an independent prognostic factor [35]. To our knowledge, these are the only available data concerning hERG1 in GC addressing hERG1 as a negative prognostic factor. On the contrary when performing a study in a larger cohort of 524 GC patients (manuscript in preparation) encompassing different stages of the disease, we obtained different conclusions. In particular, our data confirmed that hERG1 is an independent prognostic

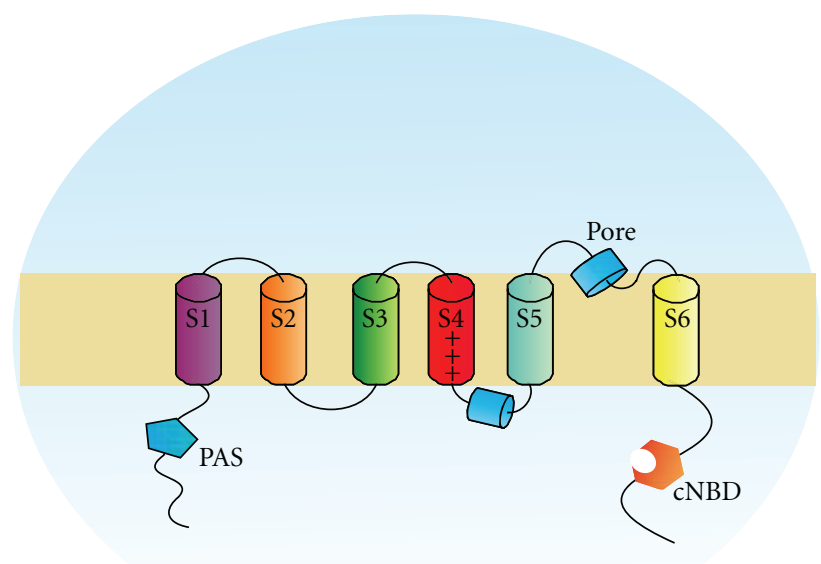

FIgure 5: hERG1 potassium channel structure; PAS: Per Arnt Sim domain, cNBD: cyclic nucleotide-binding domain.

factor, but we demonstrated its association with positive prognosis.

\section{Concluding Remarks}

Histopathology still represents the most powerful tool for gastric cancer management and, in recent years, novel biomarkers have been identified and tested for their correlations with clinical parameters as well as prognosis. In the near future new markers will be certainly validated, and the use of genomics and proteomics might help greatly clinicians in cancer management. Anyway, the possibility of validating potential tumor markers using IHC has clear advantages as it is easy and cost effective and virtually every pathology laboratory could perform it. Taking into account the importance and the usefulness of IHC markers, it will be of great importance in the next future to keep on searching for novel biomarkers as well as validating those already identified.

\section{Authors' Contribution}

E. Lastraioli and M. R. Romoli contributed equally to the work.

\section{Acknowledgments}

This work was supported by Associazione Italiana per la Ricerca sul Cancro (AIRC, Grant no. 1662) and Istituto Toscano Tumori (ITT, DD Regione Toscana no. 6888) to A. Arcangeli. The authors thank Oscar Campos for helping in the execution of drawings.

\section{References}

[1] P. Bertuccio, L. Chatenoud, F. Levi et al., "Recent patterns in gastric cancer: a global overview," International Journal of Cancer, vol. 125, no. 3, pp. 666-673, 2009. 
[2] A. Jemal, R. Siegel, J. Xu, and E. Ward, "Cancer statistics, 2010," CA Cancer Journal for Clinicians, vol. 60, no. 5, pp. 277$300,2010$.

[3] J. Chen, X. L. Bu, Q. Y. Wang, P. J. Hu, and M. H. Chen, "Decreasing seroprevalence of Helicobacter pylori infection during 1993-2003 in Guangzhou, southern China," Helicobacter, vol. 12, no. 2, pp. 164-169, 2007.

[4] E. Kawakami, R. S. Machado, S. K. Ogata, and M. Langner, "Decrease in prevalence of Helicobacter pylori infection during a 10-year period in Brazilian children," Arquivos de Gastroenterologia, vol. 45, no. 2, pp. 147-151, 2008.

[5] M. A. Tkachenko, N. Z. Zhannat, L. V. Erman et al., "Dramatic changes in the prevalence of Helicobacter pylori infection during childhood: a 10-year follow-up study in Russia," Journal of Pediatric Gastroenterology and Nutrition, vol. 45, no. 4, pp. 428-432, 2007.

[6] P. Lauren, "The two histological main types of gastric carcinoma: diffuse and so-called intestinal type carcinoma," Acta Pathologica et Microbiologica Scandinavica, vol. 64, pp. 31-49, 1965.

[7] P. Correa, "Human gastric carcinogenesis: a multistep and multifactorial process-First American Cancer Society Award lecture on cancer epidemiology and prevention," Cancer Research, vol. 52, no. 24, pp. 6735-6740, 1992.

[8] F. Carneiro, D. G. Huntsman, T. C. Smyrk et al., "Model of the early development of diffuse gastric cancer in E-cadherin mutation carriers and its implications for patient screening," Journal of Pathology, vol. 203, no. 2, pp. 681-687, 2004.

[9] W. K. Leung and J. J. Y. Sung, "Review article: intestinal metaplasia and gastric carcinogenesis," Alimentary Pharmacology and Therapeutics, vol. 16, no. 7, pp. 1209-1216, 2002.

[10] B. Mayer, J. P. Johnson, F. Leitl et al., "E-cadherin expression in primary and metastatic gastric cancer: down-regulation correlates with cellular dedifferentiation and glandular disintegration," Cancer Research, vol. 53, no. 7, pp. 1690-1695, 1993.

[11] J. C. Machado, F. Carneiro, S. Beck et al., "E-cadherin expression is correlated with the isolated cell/diffuse histotype and with features of biological aggressiveness of gastric carcinoma," International Journal of Surgical Pathology, vol. 6, no. 3, pp. 135-144, 1998.

[12] A. Bremm, A. Walch, M. Fuchs et al., "Enhanced activation of epidermal growth factor receptor caused by tumor-derived Ecadherin mutations," Cancer Research, vol. 68, no. 3, pp. 707714, 2008.

[13] J. Teruya-Feldstein, "The immunohistochemistry laboratory looking at molecules and preparing for tomorrow," Archives of Pathology and Laboratory Medicine, vol. 134, no. 11, pp. 1659$1665,2010$.

[14] N. Normanno, A. De Luca, C. Bianco et al., "Epidermal growth factor receptor (EGFR) signaling in cancer," Gene, vol. 366, no. 1, pp. 2-16, 2006.

[15] L. Fornaro, M. Lucchesi, C. Caparello et al., "Anti-HER agents in gastric cancer: from bench to bedside," Nature Reviews Gastroenterology and Hepatology, vol. 8, no. 7, pp. 369-383, 2011.

[16] S. Ménard, E. Tagliabue, M. Campiglio, and S. M. Pupa, "Role of HER2 gene overexpression in breast carcinoma," Journal of Cellular Physiology, vol. 182, no. 2, pp. 150-162, 2000.

[17] A. Sorkin and L. K. Goh, "Endocytosis and intracellular trafficking of ErbBs," Experimental Cell Research, vol. 314, no. 17, pp. 3093-3106, 2008.
[18] D. J. Slamon, W. Godolphin, L. A. Jones et al., "Studies of the HER-2/neu proto-oncogene in human breast and ovarian cancer," Science, vol. 244, no. 4905, pp. 707-712, 1989.

[19] G. Giuffrè, A. Ieni, V. Barresi, R. A. Caruso, and G. Tuccari, "HER2 status in unusual histological variants of gastric adenocarcinomas," Journal of Clinical Pathology, vol. 65, no. 3, pp. 237-241, 2012.

[20] X. L. Zhang, Y. S. Yang, D. P. Xu et al., "Comparative study on overexpression of Her2/Neu and Her3 in gastric cancer," World Journal of Surgery, vol. 33, no. 10, pp. 2112-2118, 2009.

[21] C. Gravalos and A. Jimeno, "HER2 in gastric cancer: a new prognostic factor and a novel therapeutic target," Annals of Oncology, vol. 19, no. 9, pp. 1523-1529, 2008.

[22] D. I. Park, J. W. Yun, J. H. Park et al., "HER-2/neu amplification is an independent prognostic factor in gastric cancer," Digestive Diseases and Sciences, vol. 51, no. 8, pp. 1371-1379, 2006.

[23] I. García, F. Vizoso, A. Martín et al., "Clinical significance of the epidermal growth factor receptor and HER2 receptor in resectable gastric cancer," Annals of Surgical Oncology, vol. 10, no. 3, pp. 234-241, 2003.

[24] E. Van Cutsem et al., "Efficacy results from the ToGA trial: a phase III study of trastuzumab added to standard chemotherapy (CT) in first-line human epidermal growth factor receptor 2 (HER2)-positive advanced gastric cancer (GC)," Journal of Clinical Oncology, vol. 27, p. 18s, 2009.

[25] G.-C. Li, Z. Zhang, X.-J. Ma, W.-L. Gu, Y.-N. Wang, and J. $\mathrm{Li}$, "Are biomarkers correlated with recurrence patterns in patients with resectable gastric adenocarcinoma," Molecular Biology Reports, vol. 39, no. 1, pp. 399-405, 2012.

[26] J. D. Barros-Silva et al., "Association of ERBB2 gene status with histopathological parameters and disease-specific survival in gastric carcinoma patients," British Journal of Cancer, vol. 100, no. 3, pp. 487-493, 2009.

[27] M. Tanner, M. Hollmén, T. T. Junttila et al., "Amplification of HER-2 in gastric carcinoma: association with Topoisomerase II $\alpha$ gene amplification, intestinal type, poor prognosis and sensitivity to trastuzumab," Annals of Oncology, vol. 16, no. 2, pp. 273-278, 2005.

[28] Z.-Q. Zhao, S. Yang, and H.-S. Lu, "Expression of midkine and vascular endothelial growth factor in gastric cancer and the association of high levels with poor prognosis and survival," Molecular Medicine Reports, vol. 5, no. 2, pp. 415-419, 2012.

[29] S. Osinsky, L. Bubnovskaya, I. Ganusevich et al., "Hypoxia, tumour-associated macrophages, microvessel density, VEGF and matrix metalloproteinases in human gastric cancer: interaction and impact on survival," Clinical and Translational Oncology, vol. 13, no. 2, pp. 133-138, 2011.

[30] X. Wang, W. Cao, M. Mo, W. Wang, H. Wu, and J. Wang, "VEGF and cortactin expression are independent predictors of tumor recurrence following curative resection of gastric cancer," Journal of Surgical Oncology, vol. 102, no. 4, pp. 325$330,2010$.

[31] Y. Zhou, "Clinicapathological significance of E-cadherin, VEGF, and MMPs in gastric cancer," Tumor Biology, vol. 31, pp. 549-558, 2010.

[32] C. N. Chen, F. J. Hsieh, Y. M. Cheng et al., "The significance of placenta growth factor in angiogenesis and clinical outcome of human gastric cancer," Cancer Letters, vol. 213, no. 1, pp. 73-82, 2004.

[33] C. C. Schimanski, F. Schlaegel, M. Jordan et al., "VEGF-D correlates with metastatic disease in gastric cancer patients undergoing surgery," World Journal of Surgery, vol. 35, no. 5, pp. 1010-1016, 2011. 
[34] A. Ohtsu, M. A. Shah, E. Van Cutsem et al., "Bevacizumab in combination with chemotherapy as first-line therapy in advanced gastric cancer: a randomized, double-blind, placebo-controlled phase III study," Journal of Clinical Oncology, vol. 29, no. 30, pp. 3968-3976, 2011.

[35] X. W. Ding, W. B. Yang, S. Gao et al., "Prognostic significance of hERG1 expression in gastric cancer," Digestive Diseases and Sciences, vol. 55, no. 4, pp. 1004-1010, 2010.

[36] M.-S. Soon, L.-S. Hsu, C.-J. Chen et al., "Expression of Kruppel-like factor 5 in gastric cancer and its clinical correlation in Taiwan," Virchows Archiv, vol. 459, no. 2, pp. 161-166, 2011.

[37] M. K. Kwak, H.-J. Lee, K. Hur et al., "Expression of Krüppellike factor 5 in human gastric carcinomas," Journal of Cancer Research and Clinical Oncology, vol. 134, no. 2, pp. 163-167, 2008.

[38] Y. Kato, M. Yashiro, S. Noda et al., "Expression of a hypoxiaassociated protein, carbonic anhydrase-9, correlates with malignant phenotypes of gastric carcinoma," Digestion, vol. 82, no. 4, pp. 246-251, 2010.

[39] G. G. Demirag, Y. Sullu, D. Gurgenyatagi, N. O. Okumus, and I. Yucel, "Expression of plakophilins (PKP1, PKP2, and PKP3) in gastric cancers," Diagnostic Pathology, vol. 6, no. 1, article 1, 2011.

[40] T. Sudo, K. Mimori, N. Nishida et al., "Histone deacetylase 1 expression in gastric cancer," Oncology Reports, vol. 26, no. 4, pp. 777-782, 2011.

[41] K. Mutze, R. Langer, K. Becker et al., "Histone deacetylase (HDAC) 1 and 2 expression and chemotherapy in gastric cancer," Annals of Surgical Oncology, vol. 17, no. 12, pp. 33363343,2010

[42] W. Weichert, A. Röske, V. Gekeler et al., "Association of patterns of class I histone deacetylase expression with patient prognosis in gastric cancer: a retrospective analysis," The Lancet Oncology, vol. 9, no. 2, pp. 139-148, 2008.

[43] L. Xu, X. Li, E. S. Chu et al. et al., "Epigenetic inactivation of BCL6B, a novel functional tumour suppressor for gastric cancer, is associated with poor survival," GUT, vol. 61, no. 7, pp. 977-985, 2012.

[44] X. Lu, C. Cheng, S. Zhu et al., "SATB1 is an independent prognostic marker for gastric cancer in a Chinese population," Oncology Reports, vol. 24, no. 4, pp. 981-987, 2010.

[45] C. Cheng, X. Lu, G. Wang et al., "Expression of SATB1 and heparanase in gastric cancer and its relationship to clinicopathologic features," Acta Pathologica, Microbiologica et Immunologica Scandinavica, vol. 118, no. 11, pp. 855-863, 2010.

[46] J. Ananiev et al., "Relation between transforming growth factor-b1 expression, its receptor and clinicopathological factors and survival in HER2-negative gastric cancers," Wiener Klinische Wochenschrift, vol. 123, pp. 668-673, 2011.

[47] F. Graziano, A. Mandolesi, A. Ruzzo et al., "Predictive and prognostic role of E-cadherin protein expression in patients with advanced gastric carcinomas treated with palliative chemotherapy," Tumor Biology, vol. 25, no. 3, pp. 106-110, 2004.

[48] F. Graziano, F. Arduini, A. Ruzzo et al., "Prognostic analysis of E-cadherin gene promoter hypermethylation in patients with surgically resected, node-positive, diffuse gastric cancer," Clinical Cancer Research, vol. 10, no. 8, pp. 2784-2789, 2004.

[49] Y. N. Zhou, C. P. Xu, B. Han et al., "Expression of E-cadherin and $\beta$-catenin in gastric carcinoma and its correlation with the clinicopathological features and patient survival," World Journal of Gastroenterology, vol. 8, no. 6, pp. 987-993, 2002.
[50] Y. E. Joo, C. S. Park, H. S. Kim, S. K. Choi, J. S. Rew, and S. J. Kim, "Prognostic significance of E-cadherin/catenin complex expression in gastric cancer," Journal of Korean Medical Science, vol. 15, no. 6, pp. 655-666, 2000.

[51] Y. Shino, A. Watanabe, Y. Yamada et al., "Clinicopathologic evaluation of immunohistochemical E-cadherin expression in human gastric carcinomas," Cancer, vol. 76, no. 11, pp. 21932201, 1995.

[52] H. E. Gabbert, W. Mueller, A. Schneiders et al., "Prognostic value of E-cadherin expression in 413 gastric carcinomas," International Journal of Cancer, vol. 69, no. 3, pp. 184-189, 1996.

[53] H. C. Chen, R. Y. Chu, P. N. Hsu et al., "Loss of E-cadherin expression correlates with poor differentiation and invasion into adjacent organs in gastric adenocarcinomas," Cancer Letters, vol. 201, no. 1, pp. 97-106, 2003.

[54] W. Yuan, Z. Chean, S. Wu et al., "Expression of EphA2 and E-cadherin in gastric cancer: correlated with tumor progression and lymphogenous metastasis," Pathology and Oncology Research, vol. 15, no. 3, pp. 473-478, 2009.

[55] C. T. Shun, M. S. Wu, J. T. Lin et al., "An immunohistochemical study of E-cadherin expression with correlations to clinicopathologioal features in gastric cancer," HepatoGastroenterology, vol. 45, no. 22, pp. 944-949, 1998.

[56] H. F. Gou et al., "Expressions of COX-2 and VEGF-C in gastric cancer: correlations with lymphoangiogenesis and prognostic implications," Journal of Experimental \& Clinical Cancer Research, vol. 30, p. 14, 2011.

[57] T. Nakao, N. Kurita, M. Komatsu et al., "Expression of thrombospondin-1 and Ski are prognostic factors in advanced gastric cancer," International Journal of Clinical Oncology, vol. 16, no. 2, pp. 145-152, 2011.

[58] S. H. Jeong, J. H. Han, J. H. Kim et al., "Bax predicts outcome in gastric cancer patients treated with 5-fluorouracil, leucovorin, and oxaliplatin palliative chemotherapy," Digestive Diseases and Sciences, vol. 56, no. 1, pp. 131-138, 2011.

[59] X. Liu, H. Cai, H. Huang, Z. Long, Y. Shi, and Y. Wang, "The prognostic significance of apoptosis-related biological markers in chinese gastric cancer patients," PLOS ONE, vol. 6, no. 12, Article ID e29670, 2011.

[60] T. Holbro, G. Civenni, and N. E. Hynes, "The ErbB receptors and their role in cancer progression," Experimental Cell Research, vol. 284, no. 1, pp. 99-110, 2003.

[61] M. Tan and D. Yu, "Molecular mechanisms of ErbB2-mediated breast cancer chemoresistance," Advances in Experimental Medicine and Biology, vol. 608, pp. 119-129, 2007.

[62] S. Uchino, H. Tsuda, K. Maruyama et al., "Overexpression of c-erbB-2 protein in gastric cancer: its correlation with longterm survival of patients," Cancer, vol. 72, no. 11, pp. 31793184, 1993.

[63] M. Nakajima, H. Sawada, Y. Yamada et al., "The prognostic significance of amplification and overexpression of c- met and c-erb B-2 in human gastric carcinomas," Cancer, vol. 85, no. 9, pp. 1894-1902, 1999.

[64] H. Allgayer, R. Babic, K. U. Gruetzner, A. Tarabichi, F. W. Schildberg, and M. M. Heiss, "c-erbB-2 is of independent prognostic relevance in gastric cancer and is associated with the expression of tumor-associated protease systems," Journal of Clinical Oncology, vol. 18, no. 11, pp. 2201-2209, 2000.

[65] J. S. Ross and M. Mulcahy, "HER2 testing in gastric/gastroesophageal junction adenocarcinomas: unique features of a familiar test," Gastrointestinal Cancer Research, vol. 4, no. 2, pp. 62-66, 2011. 
[66] J. Rüschoff, M. Dietel, G. Baretton et al., "HER2 diagnostics in gastric cancer-guideline validation and development of standardized immunohistochemical testing," Virchows Archiv, vol. 457, no. 3, pp. 299-307, 2010.

[67] M. Hofmann, O. Stoss, D. Shi et al., "Assessment of a HER2 scoring system for gastric cancer: results from a validation study," Histopathology, vol. 52, no. 7, pp. 797-805, 2008.

[68] Y. J. Bang, E. Van Cutsem, and A. Feyereislova, "Trastuzumab in combination with chemotherapy versus chemotherapy alone for treatment of HER2- positive advanced gastric or gastro-oesophageal junction cancer (ToGA): a phase 3, openlabel, randomised controlled trial," The Lancet, vol. 376, pp. 687-697, 2010.

[69] L. J. Tafe, Y. Y. Janjigian, M. Zaidinski et al., "Human epidermal growth factor receptor 2 testing in gastroesophageal cancer: correlation between immunohistochemistry and fluorescence in situ hybridization," Archives of Pathology and Laboratory Medicine, vol. 135, no. 11, pp. 1460-1465, 2011.

[70] Y. S. Park, H. S. Hwang, H. J. Park et al., "Comprehensive analysis of HER2 expression and gene amplification in gastric cancers using immunohistochemistry and in situ hybridization: which scoring system should we use?" Human Pathology, vol. 43, no. 3, pp. 413-422, 2012.

[71] C. K. Osborne, P. Neven, L. Y. Dirix et al., "Gefitinib or placebo in combination with tamoxifen in patients with hormone receptor-positive metastatic breast cancer: a randomized phase II study," Clinical Cancer Research, vol. 17, no. 5, pp. 1147-1159, 2011.

[72] J. Baselga, J. Albanell, A. Ruiz et al., "Phase II and tumor pharmacodynamic study of gefitinib in patients with advanced breast cancer," Journal of Clinical Oncology, vol. 23, no. 23, pp. 5323-5333, 2005.

[73] J. Baselga and S. M. Swain, "Novel anticancer targets: revisiting ERBB2 and discovering ERBB3," Nature Reviews Cancer, vol. 9, no. 7, pp. 463-475, 2009.

[74] T. Frogne, R. V. Benjaminsen, K. Sonne-Hansen et al., "Activation of ErbB3, EGFR and Erk is essential for growth of human breast cancer cell lines with acquired resistance to fulvestrant," Breast Cancer Research and Treatment, vol. 114, no. 2, pp. 263-275, 2009.

[75] N. Ferrara, "Vascular endothelial growth factor," European Journal of Cancer Part A, vol. 32, no. 14, pp. 2413-2422, 1996.

[76] C. Ulyatt, J. Walker, and S. Ponnambalam, "Hypoxia differentially regulates VEGFR1 and VEGFR2 levels and alters intracellular signaling and cell migration in endothelial cells," Biochemical and Biophysical Research Communications, vol. 404, no. 3, pp. 774-779, 2011.

[77] K. Kondo, T. Kaneko, M. Baba, and H. Konno, "VEGF-C and VEGF-A synergistically enhance lymph node metastasis of gastric cancer," Biological and Pharmaceutical Bulletin, vol. 30, no. 4, pp. 633-637, 2007.

[78] S. Iordache, A. Saftoiu, C. V. Georgescu et al., "Vascular endothelial growth factor expression and microvessel density - two useful tools for the assessment of prognosis and survival in gastric cancer patients," Journal of Gastrointestinal and Liver Diseases, vol. 19, no. 2, pp. 135-139, 2010.

[79] P. Aurello, R. Bellagamba, S. R. Del Monte et al., "Apoptosis and microvessel density in gastric cancer: correlation with tumor stage and prognosis," American Surgeon, vol. 75, no. 12, pp. 1183-1188, 2009.

[80] K. Maeda, S.-M. Kang, N. Onoda et al., "Vascular endothelial growth factor expression in preoperative biopsy specimens correlates with disease recurrence in patients with early gastric carcinoma," Cancer, vol. 86, no. 4, pp. 566-571, 1999.
[81] N. Boku, A. Ohtsu, F. Nagashima, K. Shirao, and W. Koizumi, "Relationship between expression of vascular endothelial growth factor in tumor tissue from gastric cancers and chemotherapy effects: comparison between S-1 alone and the combination of S-1 plus CDDP," Japanese Journal of Clinical Oncology, vol. 37, no. 7, pp. 509-514, 2007.

[82] J. H. Morals Cabral, A. Lee, S. L. Cohen, B. T. Chait, M. Li, and R. Mackinnon, "Crystal structure and functional analysis of the HERG potassium channel $\mathrm{N}$ terminus: a eukaryotic PAS domain," Cell, vol. 95, no. 5, pp. 649-655, 1998.

[83] M. C. Sanguinetti and M. Tristani-Firouzi, "hERG potassium channels and cardiac arrhythmia," Nature, vol. 440, no. 7083, pp. 463-469, 2006.

[84] A. Masi, A. Becchetti, R. Restano-Cassulini et al., "hERG1 channels are overexpressed in glioblastoma multiforme and modulate VEGF secretion in glioblastoma cell lines," British Journal of Cancer, vol. 93, no. 7, pp. 781-792, 2005.

[85] A. Arcangeli, "Expression and role of hERG channels in cancer cells," Novartis Foundation Symposium, vol. 266, pp. 225-232, 2005.

[86] J. Jehle, P. A. Schweizer, H. A. Katus, and D. Thomas, "Novel roles for hERG $\mathrm{K}^{+}$channels in cell proliferation and apoptosis," Cell Death and Disease, vol. 2, no. 8, Article ID e193, 2011.

[87] A. Arcangeli and A. Becchetti, "Complex functional interaction between integrin receptors and ion channels," Trends in Cell Biology, vol. 16, no. 12, pp. 631-639, 2006.

[88] L. Bianchi, B. Wible, A. Arcangeli et al., "herg encodes a K+ current highly conserved in tumors of different histogenesis: a selective advantage for cancer cells?" Cancer Research, vol. 58, no. 4, pp. 815-822, 1998.

[89] A. Cherubini, G. L. Taddei, O. Crociani et al., "HERG potassium channels are more frequently expressed in human endometrial cancer as compared to non-cancerous endometrium," British Journal of Cancer, vol. 83, no. 12, pp. 1722-1729, 2000.

[90] S. Pillozzi, M. F. Brizzi, P. A. Bernabei et al., "VEGFR-1 (FLT1), $\beta 1$ integrin, and hERG $\mathrm{K}+$ channel for a macromolecular signaling complex in acute myeloid leukemia: role in cell migration and clinical outcome," Blood, vol. 110, no. 4, pp. 1238-1250, 2007.

[91] E. Lastraioli, L. Bencini, E. Bianchini et al., "hERG1 channels and Glut-1 as independent prognostic indicators of worse outcome in stage I and II colorectal cancer: a pilot study," Translational Oncology, vol. 5, no. 2, pp. 105-112, 2012.

[92] E. Lastraioli, L. Guasti, O. Crociani et al., "herg1 gene and HERG1 protein are overexpressed in colorectal cancers and regulate cell invasion of tumor cells," Cancer Research, vol. 64, no. 2, pp. 606-611, 2004.

[93] E. Lastraioli, A. Taddei, L. Messerini et al., "hERG1 channels in human esophagus: evidence for their aberrant expression in the malignant progression of Barrett's esophagus," Journal of Cellular Physiology, vol. 209, no. 2, pp. 398-404, 2006.

[94] L. Fontana, M. D’Amico, O. Crociani et al., "Long-term modulation of HERG channel gating in hypoxia," Biochemical and Biophysical Research Communications, vol. 286, no. 5, pp. 857-862, 2001.

[95] X. D. Shao, K. C. Wu, Z. M. Hao, L. Hong, J. Zhang, and D. M. Fan, "The potent inhibitory effects of cisapride, a specific blocker for human ether-a-go-go-related gene (HERG) channel, on gastric cancer cells," Cancer Biology and Therapy, vol. 4, no. 3, pp. 295-301, 2005. 
[96] E. Lastraioli et al., "hERG1 channels are overexpressed in human gastric cancer and their activity regulates cell proliferation: a novel prognostic and therapeutic target?" in Proceedings of the 6th IGCC, pp. 151-154, Yokohama, Japan, 2005.

[97] X. D. Shao, K. C. Wu, X. Z. Guo, M. J. Xie, J. Zhang, and D. M. Fan, "Expression and significance of HERG protein in gastric cancer," Cancer Biology and Therapy, vol. 7, no. 1, pp. 45-50, 2008. 


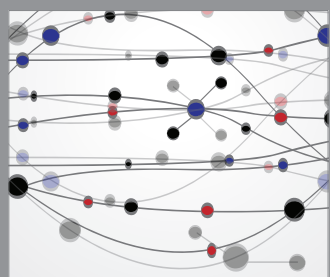

The Scientific World Journal
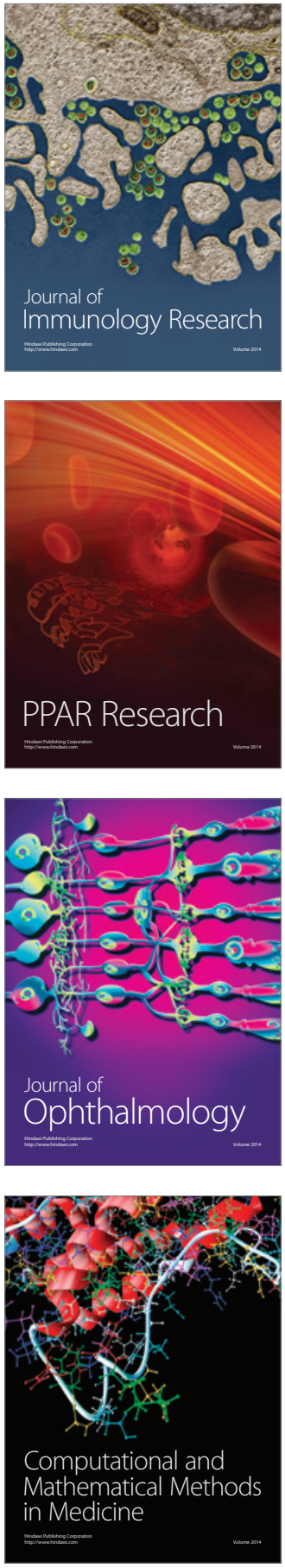

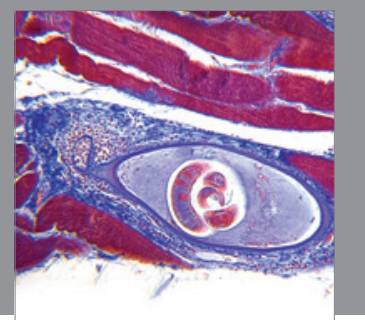

Gastroenterology

Research and Practice
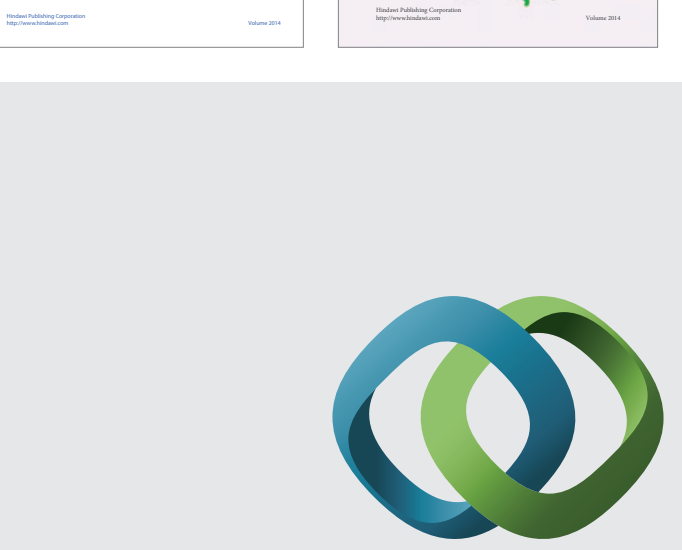

\section{Hindawi}

Submit your manuscripts at

http://www.hindawi.com
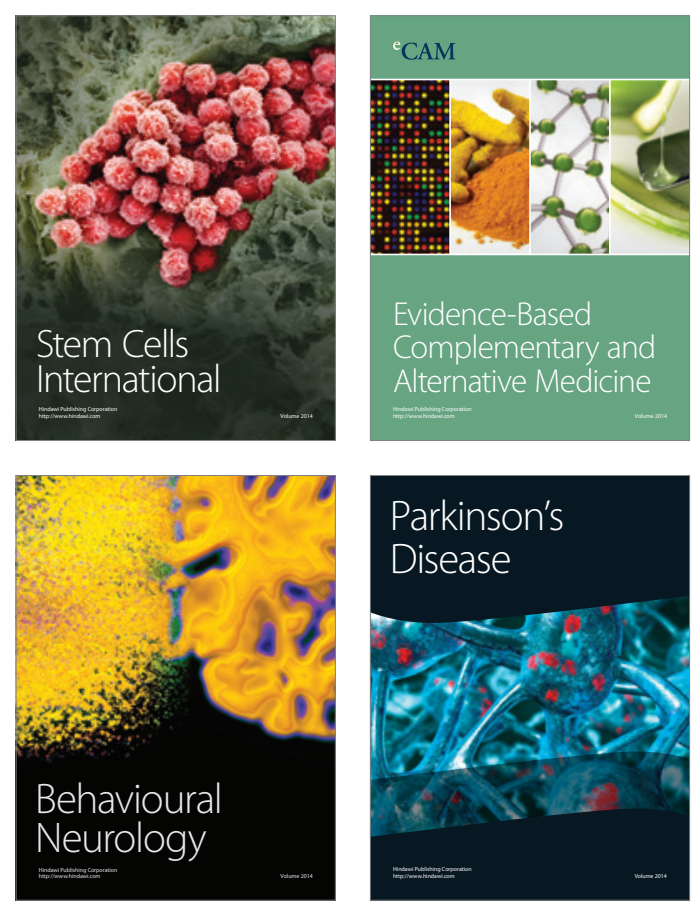

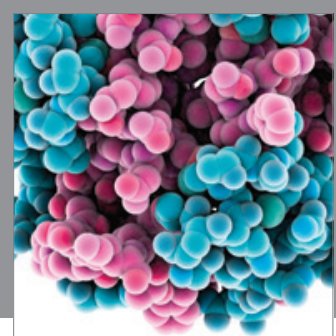

Journal of
Diabetes Research

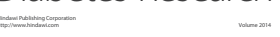

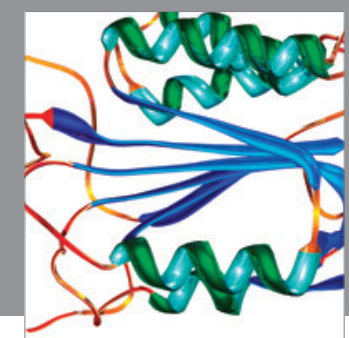

Disease Markers
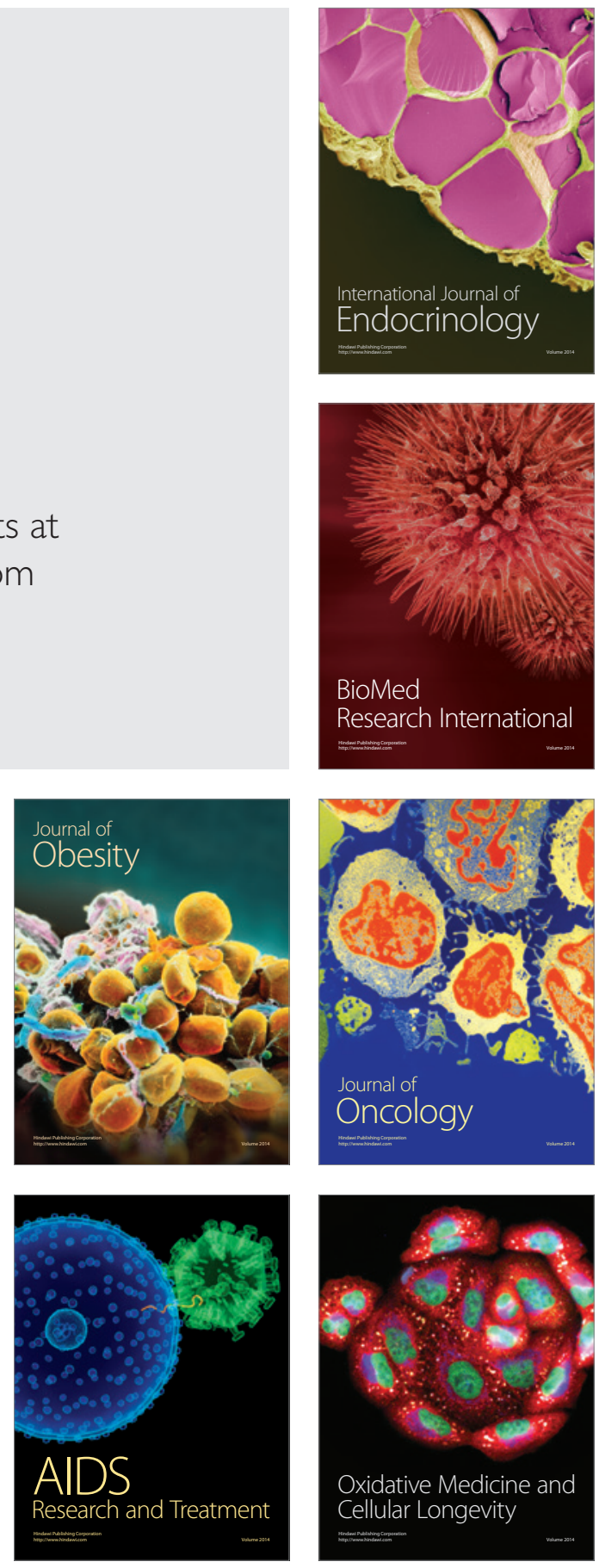